\title{
Superior parietal lobule approach for choroid plexus papillomas without preoperative embolization in very young children
}

\author{
Benjamin C. Kennedy, MD, ${ }^{1}$ Michael B. Cloney, BA, ${ }^{1}$ Richard C. E. Anderson, MD, ${ }^{1,2}$ and \\ Neil A. Feldstein, MD1,2 \\ 1'Department of Neurological Surgery and ${ }^{2}$ Children's Hospital of New York, Columbia University, New York, New York
}

OBJECT Choroid plexus papillomas (CPPs) are rare neoplasms, often found in the atrium of the lateral ventricle of infants, and cause overproduction hydrocephalus. The extensive vascularity and medially located blood supply of these tumors, coupled with the young age of the patients, can make prevention of blood loss challenging. Preoperative embolization has been advocated to reduce blood loss and prevent the need for transfusion, but this mandates radiation exposure and the additional risks of vessel injury and stroke. For these reasons, the authors present their experience using the superior parietal lobule approach to CPPs of the atrium without adjunct therapy.

METHODS A retrospective review was conducted of all children who presented to Columbia University/Morgan Stanley Children's Hospital of New York with a CPP in the atrium of the lateral ventricle and who underwent surgery using a superior parietal lobule approach without preoperative embolization.

RESULTS Nine children were included, with a median age of 7 months. There were no perioperative complications or new neurological deficits. All patients had intraoperative blood loss of less than $100 \mathrm{ml}$, with a mean minimum hematocrit of $26.9 \%$ (range $19.6 \%-36.2 \%$ ). No patients required a blood transfusion. The median follow-up was 39 months, during which time no patient demonstrated residual or recurrent tumor on MRI, nor did any have an increase in ventricular size or require CSF diversion.

CONCLUSIONS The superior parietal lobule approach is safe and effective for very young children with CPPs in the atrium of the lateral ventricle. The results suggest that preoperative embolization is not essential to avoid transfusion or achieve overall good outcomes in these patients. This management strategy avoids radiation exposure and the additional risks associated with embolization.

http://thejns.org/doi/abs/10.3171/2014.11.PEDS14281

KEY WORDS choroid plexus papilloma; superior parietal lobule approach; embolization; children; pediatrics; technique

$\mathrm{C}$ HOROID plexus papillomas (CPPs) are rare, benign tumors that disproportionately affect children, particularly infants, and commonly arise in the atrium of the lateral ventricle. ${ }^{9,12}$ The rarity of this lesion means that little evidence exists for its optimal treatment. The existing literature has demonstrated that a greater extent of resection portends a better prognosis. . $9,15,17-19,21,24,27-29$ Moreover, when a subtotal resection is achieved, CPPs can subsequently undergo malignant transformation, which further emphasizes the importance of optimal surgical management. ${ }^{5}$ However, the intraventricular location of these tumors and the risk of hemorrhage due to their high vascularity and medially arising blood supply can make gross-total resection challenging, with associated morbidity and mortality. 1,3,7,9-12,16,20,23

A variety of surgical corridors have been advocated for the resection of atrial CPPs and have included the adjunct use of neuroendoscopy. Preoperative chemotherapy or em- 
bolization has been described as a strategy for reducing tumor size or reducing the tumors' propensities to bleed, respectively. 4,11,18,19,22,27 However, each operative approach, as well as any adjunct therapy, has potential complications, so it is essential that methodologies and their outcomes be studied as rigorously as possible despite the rarity of this pathology. It is with this rationale that we sought to examine the outcomes of patients whose tumors are surgically treated with the superior parietal lobule approach without adjunct therapy.

\section{Methods}

We conducted a retrospective review of all 9 children who presented to Columbia University/Morgan Stanley Children's Hospital of New York with a tumor in the atrium of the lateral ventricle between 1998 and 2012 that was later confirmed histologically to be WHO Grade I CPP. Inpatient and outpatient charts were reviewed, including clinical notes, operative notes, laboratory findings, and vital signs as well as pre- and postoperative images and pathology reports. All patients underwent open surgery via a superior parietal lobule approach, and neither an endoscope nor adjunct therapy was used.

Generally, patients underwent volumetric contrastenhanced MRI, and stereotaxy was employed. General anesthesia was induced and antiepileptic medications, dexamethasone, and antibiotics were administered. Most patients were placed in a supine position, a shoulder roll positioned contralateral to the lesion, and the patient's head turned toward the side of the lesion to allow the surgeon access to the superior parietal lobule. Some patients were placed in the lateral position, and 1 was prone with the head slightly turned. For very young patients their heads were placed on horseshoe cerebellar headrests, whereas for older patients their heads were placed in Mayfield three-point fixation. Using stereotaxy, the specifics of the incision, craniotomy, and corticectomy were chosen to optimize a favorable approach through the superior parietal lobule toward each tumor's vascular pedicle. Once the ventricle was entered, the operating microscope was used to resect the tumor. To reach the vascular pedicle as directly as possible while avoiding the bleeding that can result from excessive manipulation of tumor, the dorsal aspect of the tumor was cauterized and shrunk superficially just enough to allow visualization of the pedicle over the superior side of the tumor. Beginning at this point, the extent of the vascular pedicles were cauterized and divided along the choroid fissure of the atrium, body, and temporal horn of the ventricle as necessary, and cautery was used for intermittent shrinking of the tumor to allow better visualization and maneuverability. The devascularized tumors were then removed in a piecemeal fashion. An external ventricular drain was left in place in all patients for 2-3 days to assist wound healing, document normal pressures, and document lack of growing ventricles on postoperative MRI. Meticulous hemostasis was then obtained, and the wound was closed in layers in routine fashion. Primary dural closure was performed without a dural graft. The patients recovered in the intensive care unit, and all drains were removed at the bedside within the first 3 postopera- tive days. The surgical corridor in Patient 2, a 3-month-old boy with a $5.2 \times 5.0 \times 4.1-\mathrm{cm}$ tumor, is demonstrated in Fig. 1.

\section{Results}

\section{Presentation}

Nine patients ( 5 girls and 4 boys) were treated during the study period, and the median age was 7 months (range 2-112 months). The most common presenting symptoms or signs were bulging fontanel and developmental delay, each seen in 3 patients. Nausea and vomiting were documented in 2 patients at presentation, and 2 other patients presented with abnormal head growth, with head circumference crossing percentiles. Two patients were asymptomatic: Patient 3 had hydrocephalus, and the tumor was noted on screening MRI for her Aicardi syndrome; and Patient 8 was diagnosed with the tumor on prenatal ultrasound, and though hydrocephalus was not yet apparent, the lesion was noted to grow on serial postnatal imaging, prompting surgery.

Seven of 9 tumors were left sided. One tumor was relatively small at $2.0 \times 1.1 \times 1.0 \mathrm{~cm}$, found in a 6 -year-old boy who presented with developmental delay and seizure. The remainder of the tumors measured between 4.3 and 6.3 $\mathrm{cm}$ in greatest dimension (Table 1). Figure 2 shows MR images of representative tumors from 4 patients.

\section{Complications}

There were no intraoperative complications. In the immediate postoperative period, seizures occurred in 2 patients (Table 2). In 1 patient (Patient 2) the phenytoin level was subtherapeutic. Both patients responded to administration of antiepileptic medications and remained seizure free. There were no new neurological deficits on clinical examination, but in detailed ophthalmological follow-up, 1 small extreme-lateral visual field deficit was noted.

\section{Hemodynamics}

Preoperative hematocrit was available for 8 of 9 patients, and the mean was $35.0 \%$. Estimated intraoperative blood loss was less than $100 \mathrm{ml}$ for all 9 patients. All patients had a series of postoperative blood counts, the frequency and duration of which were based on the clinical judgment of the attending pediatric neurosurgeon and pediatric critical care attending physician. The mean of all patients' postoperative hematocrit nadirs was $26.9 \%$ (range $19.6 \%-36.2 \%$ ). For the 8 of the 9 patients for whom pre- and postoperative hematocrits were available, the mean of the decreases in hematocrit was $7.2 \%$ points (range $+1.2 \%$ to $-13.8 \%$ points). Seven of these 9 nadirs occurred on the day of surgery or the day following surgery (Table 2). All patients remained hemodynamically stable throughout their hospital course, and no patient required a blood transfusion.

\section{Clinical Follow-Up}

All patients underwent postoperative clinical followup as outpatients, and the mean follow-up period was 39 months. On extended follow-up, Patient 9 demonstrated a 

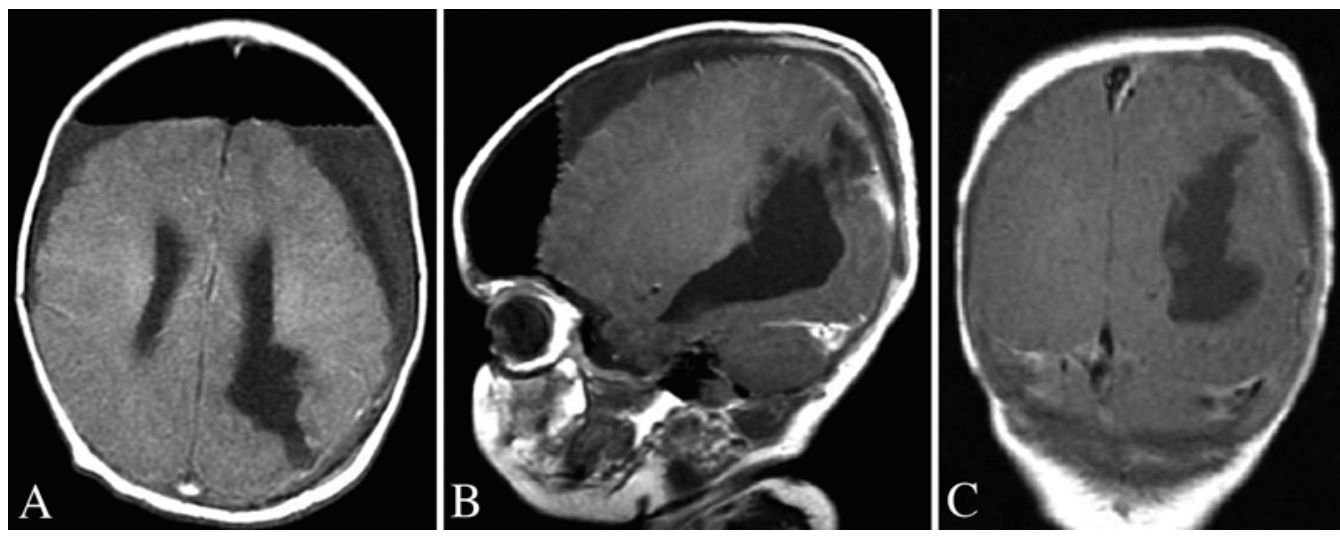

FIG. 1. Patient 2. Postoperative axial (A), sagittal (B), and coronal (C) T1-weighted postcontrast MRI scans demonstrating the operative corridor used in the superior parietal lobule approach after resection of a $5.2 \times 5.0 \times 4.1-\mathrm{cm}$ left atrial tumor in a 3-monthold boy who presented with irritability and a bulging fontanel.

small isolated extreme-lateral visual field deficit. No other neurological symptoms noted in follow-up were considered attributable to surgery but, rather, were attributed to underlying neurological disorders or prior hydrocephalus at a younger age. Patient 2 had a Dandy-Walker malformation, autism, and language problems; Patient 3 had Aicardi syndrome with resultant developmental delay; Patient 6 had attention deficit problems at 8 years of age; Patient 7 was substantially improving in his developmental delay but was still having language, balance, and chewing problems at 1 year of follow-up; and Patient 8 had mild incoordination and a lisp at 4 years of age. None of the patients with follow-up demonstrated any progressive or disabling neurological deficit (Table 2). Both infants who presented with abnormal head growth experienced a return to a normal head size and growth trajectory within 4 months of surgery. No patient required another operation for tumor or hydrocephalus. No patient underwent shunt placement.

\section{Radiographic Outcome}

Contrast-enhanced MRI was performed in 8 of 9 patients during the follow-up period, with a mean MRI follow-up of 20 months (range 2 days to 55 months) (Table 2). No patient exhibited residual or recurrent tumor, nor did any exhibit progression of hydrocephalus.

\section{Discussion}

We report our experience with 9 consecutive patients harboring CPPs in the atrium of the lateral ventricle treated with surgery alone via the superior parietal lobule approach. No patient experienced substantial intraoperative blood loss, developed hemodynamic instability, or had a hematocrit below $19.6 \%$, and no patient required a blood transfusion. No patient developed a disabling neurological deficit, demonstrated residual or recurrent tumor on MRI, or required a shunt or repeat resection. Furthermore, none of our patients were subjected to the risks inherent in angiography with embolization, radiation, or chemotherapy.

Twelve percent of brain tumors in infants are CPPs, which typically arise in the lateral ventricle of the very young and cause overproduction hydrocephalus., ${ }^{9,12,13,16,23}$ Their benign nature and intraventricular, noninfiltrative nature renders them surgically curable..$^{15,20,27}$ Gross-total resection has been associated with excellent prognosis, $, 18,20$ with 5-year survival being close to $100 \%$ after complete resection, but survival is only $56 \%$ after subtotal resection. ${ }^{6,14,15,17,19-21,24,27}$ Complete initial removal of the tumor is essential to prevent further tumor growth or recurrence, malignant degeneration, ${ }^{5}$ continued overproduction of CSF and hydrocephalus, the need for adjuvant therapies such as radiotherapy or chemotherapy, or the morbidity of repeat surgery. ${ }^{16}$

TABLE 1. Presentation and tumor characteristics

\begin{tabular}{cclll}
\hline Patient No. & Age at Op (mos), Sex & \multicolumn{1}{c}{ Presentation } & Side & Size $(\mathrm{cm})$ \\
\hline 1 & $5, \mathrm{~F}$ & Nausea/vomiting, bulging fontanel, CN VI palsy & Left & $5.2 \times 3.9 \times 3.8$ \\
\hline 2 & $3, \mathrm{M}$ & Irritability, bulging fontanel & Left & $5.2 \times 5.0 \times 4.1$ \\
\hline 3 & $15, \mathrm{~F}$ & Workup for Aicardi syndrome, hydrocephalus & Left & $5.2 \times 3.7 \times 2.5$ \\
\hline 4 & $79, \mathrm{M}$ & Developmental delay, seizure & Left & $2.0 \times 1.1 \times 1.0$ \\
\hline 5 & $4, \mathrm{~F}$ & Head growth, bulging fontanel & Right & $5.7 \times 5.5 \times 4.5$ \\
\hline 6 & $7, \mathrm{M}$ & Head growth & Left & $5.5 \times 4.9 \times 4.4$ \\
\hline 7 & $20, \mathrm{~F}$ & Developmental delay, ataxia, hypotonia & Right & $4.3 \times 3.7 \times 3.6$ \\
\hline 8 & $2, \mathrm{M}$ & Prenatal diagnosis, radiographic tumor growth & Left & $4.9 \times 4.6 \times 3.6$ \\
\hline 9 & $112, \mathrm{~F}$ & Developmental delay, headache, nausea/vomiting, papilledema & Left & $6.3 \times 5.5 \times 5.3$ \\
\hline
\end{tabular}

$\mathrm{CN}=$ cranial nerve 

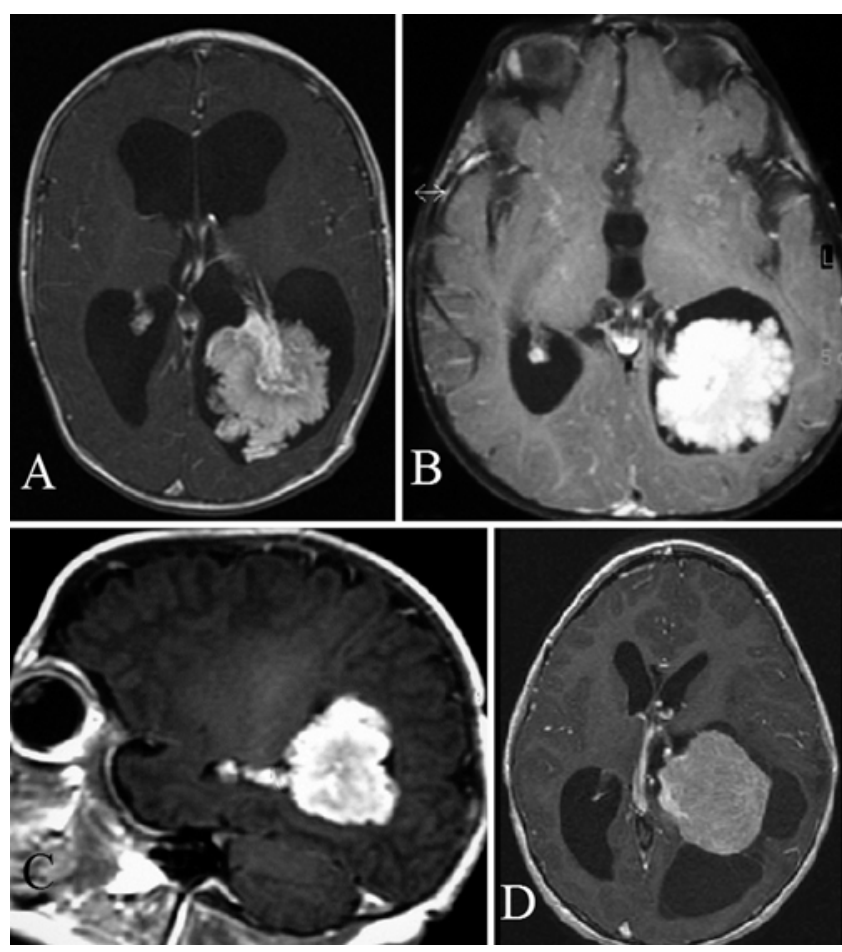

FIG. 2. Preoperative T1-weighted postcontrast MRI scans demonstrating vascular pedicles in 4 representative patients: axial study of Patient 2

(A); axial study of Patient 6 (B); sagittal study of Patient 8 (C); and axial study of Patient 9 (D).

The highly vascular nature of these lesions and frequently very small size of the patient, however, can result in clinically significant, and potentially fatal, intraoperative blood loss. . $^{1,7,10,11,20}$ One recent series documented a mean intraoperative blood loss of $182 \%$ of each patient's blood volume for unembolized CPPs..$^{11}$ In published series, the perioperative mortality rate has been reported to range from $0 \%$ to $25 \%,{ }^{3,7,9-12,16,20,23}$ and the mortality rate attributed to blood loss has been reported to be as high as $12 \% .^{12}$ In response to this difficulty, endovascular embolization has been advocated as a means to reduce intraoperative blood loss for these tumors. ${ }^{6,19,20}$ It has also been suggested that less intraoperative bleeding due to embolization leads to a higher rate of complete resection and better outcome. ${ }^{11}$
However, many series have reported a low rate of successful embolization when attempted due to small tortuous vessels that are difficult or impossible to cannulate, or feeding arteries that are judged to also perfuse deep brain structures. ${ }^{1,17,20}$ In addition to these technical difficulties, angiography and embolization are accompanied by small risks of stroke and other complications and should particularly be avoided in the very young because of both deterministic and stochastic long-term radiation effects. It has been estimated that a cerebral embolization procedure in a child may increase the relative risk of developing a brain tumor by $2 \%-80 \% .^{2,25,26}$ Other toxic treatments like radiotherapy ${ }^{12}$ and chemotherapy ${ }^{1}$ have also been described to reduce size and vascularity of these benign tumors preoperatively or to treat residual tumor postoperatively.

Blood transfusion is also not without risk, but it may be routine management at many centers for patients undergoing CPP surgery. Intraoperative or postoperative blood transfusion has been mentioned in some small series, ${ }^{18,20}$ and in 1 modern series, intraoperative and postoperative transfusion appeared to be routine management..$^{11}$ In fact, 2 of the most recent large series have each documented a mean intraoperative blood loss over $100 \%$ of each patient's total blood volume, suggesting that most patients at those centers are being transfused routinely. This, taken with the frequent discussion of blood loss and resultant death reported in the literature, suggests that blood transfusion is commonly performed in these patients, though most series do not provide detail on or discussion of the topic. The present study demonstrates that with the corridor provided by the superior parietal lobule approach to the vascular supply of these tumors, neither embolization nor transfusion is necessary in most cases to maintain normal hemodynamics in the intra- and postoperative periods.

High rates of ventriculoperitoneal ${ }^{7,15,18,20}$ or subduralperitoneal ${ }^{20}$ shunting have also been reported. Shunting is another adjunct treatment that entails well-known risks. Using our management approach, none of our patients required shunting or any other CSF diversion procedures. Our use of postoperative external ventricular drainage in selected patients may have contributed to our lack of symptomatic subdural collections, which has been suggested by the experience of other surgeons..$^{18,20}$ Beyond this, it is not clear why our shunt rate is so different from that reported

TABLE 2. Outcomes

\begin{tabular}{ccccclc}
\hline $\begin{array}{c}\text { Patient } \\
\text { No. }\end{array}$ & $\begin{array}{c}\text { Age at Op } \\
\text { (mos) }\end{array}$ & $\begin{array}{c}\text { Mean Hct } \\
\text { Nadir (\%) }\end{array}$ & $\begin{array}{c}\text { Length of } \\
\text { FU (mos) }\end{array}$ & $\begin{array}{c}\text { Periop } \\
\text { Complications }\end{array}$ & \multicolumn{1}{c}{ Neurological Outcome } & $\begin{array}{c}\text { Length of Radiographic } \\
\text { FU (mos) }\end{array}$ \\
\hline 1 & 5 & 26.6 & 12 & & No issues & 0.07 \\
\hline 2 & 3 & 26.2 & 63 & Seizure & Dandy-Walker malformation, autism & 55 \\
\hline 3 & 15 & 27.4 & 15 & & Aicardi syndrome & 15 \\
\hline 4 & 79 & 36.2 & 0.6 & & No issues & None \\
\hline 5 & 4 & 22.9 & 52 & & No issues & 14 \\
\hline 6 & 7 & 19.6 & 95 & Seizure & Attention deficit & 9 \\
\hline 8 & 20 & 31.5 & 13 & & Language \& balance problems & 13 \\
\hline 9 & 2 & 24.2 & 50 & & Incoordination & 46 \\
\hline
\end{tabular}

$\mathrm{FU}=$ follow-up; $\mathrm{Hct}=$ hematocrit. 
by other practitioners. Continued pathological hydrocephalus could be a result of persistent tumor, and our lack of shunting may be supported by our complete removal of tumor. Other authors have proposed that hydrocephalus after radiographically confirmed gross-total resection suggests that the hydrocephalus is not entirely from overproduction. ${ }^{18}$ Significant intraoperative bleeding, frequently reported in published series, can cause hydrocephalus requiring shunting, and our approach and lack of intraoperative blood loss into the ventricles may also be partly responsible for our relatively low rate of postoperative shunting. We believe that ventricles in these patients with severe hydrocephalus due to CPP from a young age will remain enlarged to some degree indefinitely and would consider shunt placement warranted if a patient were not improving clinically as expected, if an infant's head did not resume a normal growth rate, or if the patient exhibited symptoms in the follow-up period or other symptoms specifically attributable to hydrocephalus. However, considering that the rationale for shunting is not commonly discussed in the literature on these patients, attributing the different shunt rates to differing clinical philosophies remains speculative.

There are a variety of surgical corridors that can be used to approach the atrium of the lateral ventricle. A cortical incision can be made in various regions, and interhemispheric approaches are also possible. Endoscopy has also been advocated in the approach to these tumors. Highly vascular, complex intraventricular lesions, when bleeding, can be more challenging to control endoscopically, depending heavily on the experience of the surgeon. Considering the evidence supporting the importance of gross-total resection, it is essential that the approach chosen allows the surgeon to achieve this result while avoiding or maintaining control over potentially catastrophic bleeding. Even in recent series, only 54\% of unembolized CPPs were completely resected. ${ }^{11}$ The superior parietal lobule approach has been reported for CPPs of the atrium, ${ }^{8,17}$ and in the current series, it provided excellent exposure and afforded vascular control over all tumors, allowing complete resection with minimal blood loss. There are several rationales for this approach over others. First, having the lesion side of the head down and using a medial approach affords the least amount of retraction and tumor manipulation when approaching the vascular pedicle since the tumor is retracted by gravity. The superior parietal lobule approach also allows the surgeon to visualize the entirety of the vascular pedicle, from the choroidal vessels in the temporal horn to those in the body of the ventricle, as well as in the atrium, achieving complete control for any tumor, regardless of from which region or regions its blood supply comes. Most of these tumors arise on the left side, and the superior parietal lobule approach has the advantage over a temporal approach of avoiding retraction in the dominant temporal lobe for these tumors.

Limitations of this study include its retrospective nature, the small number of patients $(n=9)$, the relatively short follow-up (mean 39 months), and the fact that it is a single-surgeon series. Furthermore, we recognize that training and comfort with approaches vary substantially among neurosurgeons, and these factors should always play a large role in surgical decision making.

\section{Conclusions}

This is the first reported series of CPPs in the atria of children to demonstrate a management approach that avoids the risks and side effects of angiography, embolization, blood transfusion, radiotherapy, chemotherapy, shunt placement, and repeat surgery in all patients while achieving excellent neurological and radiological results.

\section{References}

1. Addo NK, Kamaly-Asl ID, Josan VA, Kelsey AM, Estlin EJ: Preoperative vincristine for an inoperable choroid plexus papilloma: a case discussion and review of the literature. $\mathbf{J}$ Neurosurg Pediatr 8:149-153, 2011

2. Alexander MD, Oliff MC, Olorunsola OG, Brus-Ramer M, Nickoloff EL, Meyers PM: Patient radiation exposure during diagnostic and therapeutic interventional neuroradiology procedures. J Neurointerv Surg 2:6-10, 2010

3. Berger C, Thiesse P, Lellouch-Tubiana A, Kalifa C, PierreKahn A, Bouffet E: Choroid plexus carcinomas in childhood: clinical features and prognostic factors. Neurosurgery 42:470-475, 1998

4. Cappabianca P, Cinalli G, Gangemi M, Brunori A, Cavallo LM, de Divitiis E, et al: Application of neuroendoscopy to intraventricular lesions. Neurosurgery 62 (Suppl 2):575598,2008

5. Chow E, Reardon DA, Shah AB, Jenkins JJ, Langston J, Heideman RL, et al: Pediatric choroid plexus neoplasms. Int J Radiat Oncol Biol Phys 44:249-254, 1999

6. Do HM, Marx WF, Khanam H, Jensen ME: Choroid plexus papilloma of the third ventricle: angiography, preoperative embolization, and histology. Neuroradiology 43:503-506, 2001

7. Due-Tønnessen B, Helseth E, Skullerud K, Lundar T: Choroid plexus tumors in children and young adults: report of 16 consecutive cases. Childs Nerv Syst 17:252-256, 2001

8. Ellenbogen RG: Transcortical surgery for lateral ventricular tumors. Neurosurg Focus 10(6):E2, 2001

9. Ellenbogen RG, Winston KR, Kupsky WJ: Tumors of the choroid plexus in children. Neurosurgery 25:327-335, 1989

10. Guidetti B, Spallone A: The surgical treatment of choroid plexus papillomas: the results of 27 years experience. Neurosurg Rev 4:129-137, 1981

11. Haliasos N, Brew S, Robertson F, Hayward R, Thompson D, Chakraborty A: Preoperative embolisation of choroid plexus tumours in children: part I-does the reduction of perioperative blood loss affect the safety of subsequent surgery? Childs Nerv Syst 29:65-70, 2013

12. Hawkins JC III: Treatment of choroid plexus papillomas in children: a brief analysis of twenty years' experience. Neurosurgery 6:380-384, 1980

13. Jooma R, Hayward RD, Grant DN: Intracranial neoplasms during the first year of life: analysis of one hundred consecutive cases. Neurosurgery 14:31-41, 1984

14. Lafay-Cousin L, Keene D, Carret AS, Fryer C, Brossard J, Crooks B, et al: Choroid plexus tumors in children less than 36 months: the Canadian Pediatric Brain Tumor Consortium (CPBTC) experience. Childs Nerv Syst 27:259-264, 2011

15. Lena G, Genitori L, Molina J, Legatte JR, Choux M: Choroid plexus tumours in children. Review of 24 cases. Acta Neurochir (Wien) 106:68-72, 1990

16. McGirr SJ, Ebersold MJ, Scheithauer BW, Quast LM, Shaw EG: Choroid plexus papillomas: long-term follow-up results in a surgically treated series. J Neurosurg 69:843-849, 1988

17. Nagib MG, O'Fallon MT: Lateral ventricle choroid plexus papilloma in childhood: management and complications. Surg Neurol 54:366-372, 2000

18. Ogiwara H, Dipatri AJ Jr, Alden TD, Bowman RM, Tomita 
T: Choroid plexus tumors in pediatric patients. Br J Neurosurg 26:32-37, 2012

19. Otten ML, Riina HA, Gobin YP, Souweidane MM: Preoperative embolization in the treatment of choroid plexus papilloma in an infant. Case report. J Neurosurg 104 (6 Suppl):419-421, 2006

20. Pencalet P, Sainte-Rose C, Lellouch-Tubiana A, Kalifa C, Brunelle F, Sgouros S, et al: Papillomas and carcinomas of the choroid plexus in children. J Neurosurg 88:521-528, 1998

21. Raimondi AJ, Gutierrez FA: Diagnosis and surgical treatment of choroid plexus papillomas. Childs Brain 1:81-115, 1975

22. Souweidane MM, Johnson JH Jr, Lis E: Volumetric reduction of a choroid plexus carcinoma using preoperative chemotherapy. J Neurooncol 43:167-171, 1999

23. Spallone A, Pastore FS, Giuffre R, Guidetti B: Choroid plexus papillomas in infancy and childhood. Childs Nerv Syst 6:71-74, 1990

24. Strojan P, Popović M, Surlan K, Jereb B: Choroid plexus tumors: a review of 28-year experience. Neoplasma 51:306312,2004

25. Thierry-Chef I, Simon SL, Land CE, Miller DL: Radiation dose to the brain and subsequent risk of developing brain tumors in pediatric patients undergoing interventional neuroradiology procedures. Radiat Res 170:553-565, 2008

26. Thierry-Chef I, Simon SL, Miller DL: Radiation dose and cancer risk among pediatric patients undergoing interventional neuroradiology procedures. Pediatr Radiol 36 (Suppl 2):159-162, 2006

27. Wolff JE, Sajedi M, Brant R, Coppes MJ, Egeler RM: Choroid plexus tumours. Br J Cancer 87:1086-1091, 2002
28. Wolff JE, Sajedi M, Coppes MJ, Anderson RA, Egeler RM: Radiation therapy and survival in choroid plexus carcinoma. Lancet 353:2126, 1999 (Letter)

29. Wrede B, Liu P, Ater J, Wolff JE: Second surgery and the prognosis of choroid plexus carcinoma-results of a metaanalysis of individual cases. Anticancer Res 25 (6C):44294433, 2005

\section{Author Contributions}

Conception and design: Kennedy, Anderson, Feldstein. Acquisition of data: Kennedy, Cloney, Feldstein. Analysis and interpretation of data: all authors. Drafting the article: Kennedy, Anderson, Feldstein. Critically revising the article: Kennedy, Anderson, Feldstein. Reviewed submitted version of manuscript: all authors. Approved the final version of the manuscript on behalf of all authors: Kennedy. Study supervision: Feldstein.

\section{Supplemental Information}

\section{Previous Presentation}

A version of this work was presented in digital poster form at the 2014 Congress of Neurological Surgeons Annual Meeting, October 18-22, 2014, Boston, Massachusetts.

\section{Correspondence}

Benjamin C. Kennedy, The Neurological Institute, 710 W. 168th St., 4th Fl., New York, NY 10032. email: bck2104@ columbia. edu. 